\title{
Smart Mirror
}

\section{Shreyansh Khale, Aditi Sathe, Rugveda Salunke, Shweta Nathan, Amit Maurya}

\begin{abstract}
This paper describes and explains the design and working of a mirror made smart called Smart mirror built using raspberry pi. The visual representation of the product is similar to a regular mirror that can display weather details, temperature, time and daily news on voice commands. The smart mirror acts as a personal assistant, an enquiry center and displays important curriculum or college notices. It can answer basic questions, display class timetable and show directions to various places of an institute. It could be linked to google cloud for various IOT based tasks like home automation etc. It uses a proximity sensor to turn on the screen if in use and turn back off when not in use. Built on a strong speech to text engine, it understands various types of accents to understand predefined command clearly. The smart or interactive mirror designed by using a raspberry pi as the main controller and a led display that is placed behind the mirror, can serve a variety of endless application-based commands. With such a wide range of applications, this could certainly become an important part of technology in future times.
\end{abstract}

Keywords: Smart mirror, notice board, personal assistant, enquiry center.

\section{INTRODUCTION}

Mirrors are part of everyday used items that have been so far eluded from the idea of being smart. The idea of combination of mirror with intelligence and technology, discussing further possibilities and uses are some objectives of this paper. The smart mirror may seem to be similar to that of various other smart devices available like smart phones, smart televisions, smart lights etc. but have some certain and specific advantages because of its usage as a mirror. This smart mirror is a still complex to that of an ordinary mirror, having a display inside a glass that one can interact with, using voice commands.

The mirror works with the help of a raspberry pi. There are several displays or notice boards present that are difficult to operate manually. The problem of finding places in new buildings were too a concern. This product acts as a solution for above problems. It also acts as an innovative and attractive object that can be placed in any surrounding.

A product that can inhibit all the qualities of a regular personal assistant on androids or laptops with

Revised Version Manuscript Received on 16 September, 2019.

Shreyansh Khale, Mumbai University, Vidyalankar Institute of Technology (VIT), Wadala, Mumbai, Maharashtra, India.

(Email: khaleshreyansh@gmail.com)

Aditi Sathe, Mumbai University, Vidyalankar Institute of Technology

(VIT), Wadala, Mumbai, Maharashtra, India.

(Email: aditiss14@gmail.com)

Rugveda Salunke, Mumbai University, Vidyalankar Institute of

Technology (VIT), Wadala, Mumbai, Maharashtra, India.

(Email: rugveda2604@gmail.com)

Shweta Nathan, Mumbai University, Vidyalankar Institute of Technology (VIT), Wadala, Mumbai, Maharashtra, India.

(Email: shwetanathan18@gmail.com,)

Amit Maurya, Mumbai University, Vidyalankar Institute of Technology

(VIT), Wadala, Mumbai, Maharashtra, India.

(Email: amit.maurya@ vit.edu.in) application-based knowledge along with the above-mentioned problems with some suitable speech detection modules and speech convertor engines, all in a small processing power operating system as that of a raspberry pi (Debian).

Putting the tasks above in a simplified manner, three basic functionalities that would be fulfilled can be given as,

\section{Personal Assistant-}

The mirror acts like a personal assistant. The mirror does various tasks like updating calendar, setting up reminders, updating date and time, displaying weather, daily news and other such general-purpose activities. It can respond to some of the commands like time, weather, news, Gmail, technology updates, college notifications, birthday, jokes, life.

\section{Enquiry Centre-}

Basic questions related to any workspace are fed into the database. Such queries can be answered by the mirror. This enquiry center can be very helpful to people who are new to any workspace. These queries will be regarding finding the direction to any particular classroom, locating any professor etc. This can be an endless module as we can configure as many questions as we need. Thus, this can differ based on the workspace in which you are using this mirror.

\section{Notice board-}

The mirror can be used for displaying various academic notices in the college. The product is linked with the android device of the administrator so that he can change the notice and information as and when required to be displayed. These notices are displayed according to various time slots.

\section{LITERATURE SURVEY}

Michael Teeuw's [1] was the first to build a smart mirror and first to use a raspberry pi for this purpose. The first smart mirror blog was posted back in 2014, since it was a very new product it gained a lot of attention back then. This mirror is built on raspberry pi 2 and uses monitor as the display. It displayed weather and time importing these from various modules which were linked to real time websites. It was just an information panel which didn't have the capability to interact with the mirror. A module-based interface was created and displayed weather, news, time or daily comic strip.

Ryan Nelwan [2] in the year 2016 gathered much interest and developed a smart mirror much similar to the one developed by teeuw's. A new feature added to this was the $t$

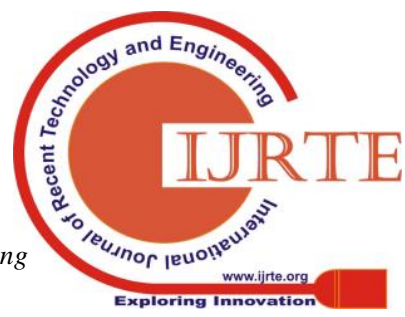


ouch feature which was a first of its kind. It serves mostly as a source of a entertainment system in which a user can use the touch controls to run different programs or control music, but did not have artificial intelligence.

Hannah Mittelstaedt [3] made a home mirror. It was posted on reddit website. The mirror used a smart phone as the display screen. Since it was an android tablet so features of android were used to display time, weather, date, remainders. The software made use of android widgets but can be modified easily as it is open source. Anyone can modify it and develop a new version. Home Mirror is a kind of smart mirror that is easier to build than other mirrors as it requires just two main components, any android mobile phone or a tablet and a mirror. However, this too lacked any kind of intelligence or interaction.

\section{SYSTEM DESIGN \& RESULTS}

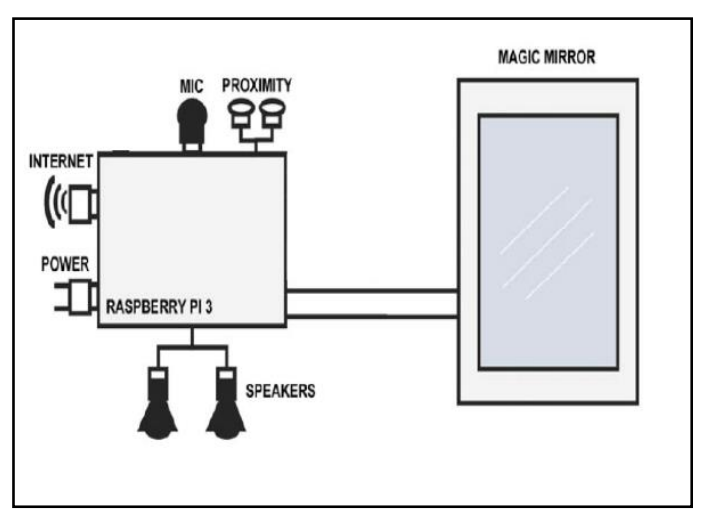

Figure 1-Block diagram

The software structure of the system is implemented and built using the web socket communication protocol. The architecture is modular and works on the basis of function designated modules which can be incorporated together on a single platform. With this, we can club many features such as clock, calendar, current weather, newsfeed etc., and many other third-party functionalities. The server module leads all the operations: interaction with the user through the sensors and the mirror user interface (UI), information visualization through the mirror UI etc.

\section{A. Notice:}

This module displays a random notice regarding any academic activities that are to be conducted in the college. The notification property contains an object with four arrays: 'morning', 'afternoon', 'evening' and 'anytime'.

\section{B. Newsfeed:}

This module displays news headlines based on a really simple syndication (RSS) feed. Scrolling through news headlines happens time-based (update Interval), but can also be controlled by sending news feed specific notifications to the module. An array of feed URLs are used as source. The URL of the feed is used for headlines.

\section{Update Notification:}

This will display a message whenever a new version of the Magic Mirror application is available.

\section{D.Clock:}

This module displays the current date and time. The information will be updated real time. It displays time in 24-hour time format. The time zone Asia/Kolkata is used for displaying time.

\section{E. Current Weather:}

This module displays the current weather, the sunset or sunrise time, the temperature including the windspeed and an icon to display the current conditions. The location feed for the weather information is Mumbai, Maharashtra, India. The weather information is obtained from OpenWeatherMap. It is an online service that provides weather data, including current weather data and forecasts.

\section{F. Weather Forecast:}

This module displays the weather forecast for the coming week, including an icon to display the current conditions, the minimum temperature and the maximum temperature. The URL used for this module is same as that of current weather module.

\section{G. Weekly Schedule:}

It displays today's timetable from a weekly recurring schedule of a timetable of particular class. It is intended for regular weekly schedules, which have a low update frequency and thus can be maintained manually.

\section{H.Slide Changer:}

This is an extension to the above modules, allowing the modules to be displayed in a rotating carousel instead of displaying all of them at once.

\section{Alarm:}

In this an array is created where all the alarms are treated as objects. These objects have properties like time, 24-hour format, days etc. A sound is set for the alarm and if the sound is not defined alarm will be fired with default alarm sound.

Along with these certain voice modules can be included which are accessible through voice commands. They are:

\section{a. Technology news:}

This module gives the latest technology news when asked by the user. Technology News is a social news website that caters to programmers and entrepreneur, delivering content related to computer science and entrepreneurship. Jasper technology news will notify the user about the top 10 stories.

\section{b. Status:}

This module gives information regarding the status of the mirror like in which platform it is running, its current CPU utilization, current memory utilization etc.

\section{c. Message queuing telemetry transport:}

When triggered it publishes a simple message queuing telemetry transport (MQTT) event. The available devices are room, door and the available messages are on, off, true, false, open, close.

\section{d. Reboot and Shutdown:}

Used to reboot and shutdown the raspberry pi using voice.

\section{Published By:}




\section{WORKING OF PROJECT}

For the hardware architecture, a Dell computer monitor, a two-way mirror, a Raspberry $\mathrm{Pi}$ model 3B, USB microphones, jack speaker and a proximity sensor are used.

Everything was put together in a wooden frame. The entire structure is divided into two wooden parts constructed as a box type structure. The behind part holds the display screen and the Raspberry Pi and is used to support the device so that it can be hung on a wall. The forward portion of the box type structure is made using the glass which is made to fit entirely in front the screen.

The major components that are used (the two-way mirror glass, display, Raspberry $\mathrm{Pi}$, microphones, proximity sensors and frame) and how they are used is described in the following sections:

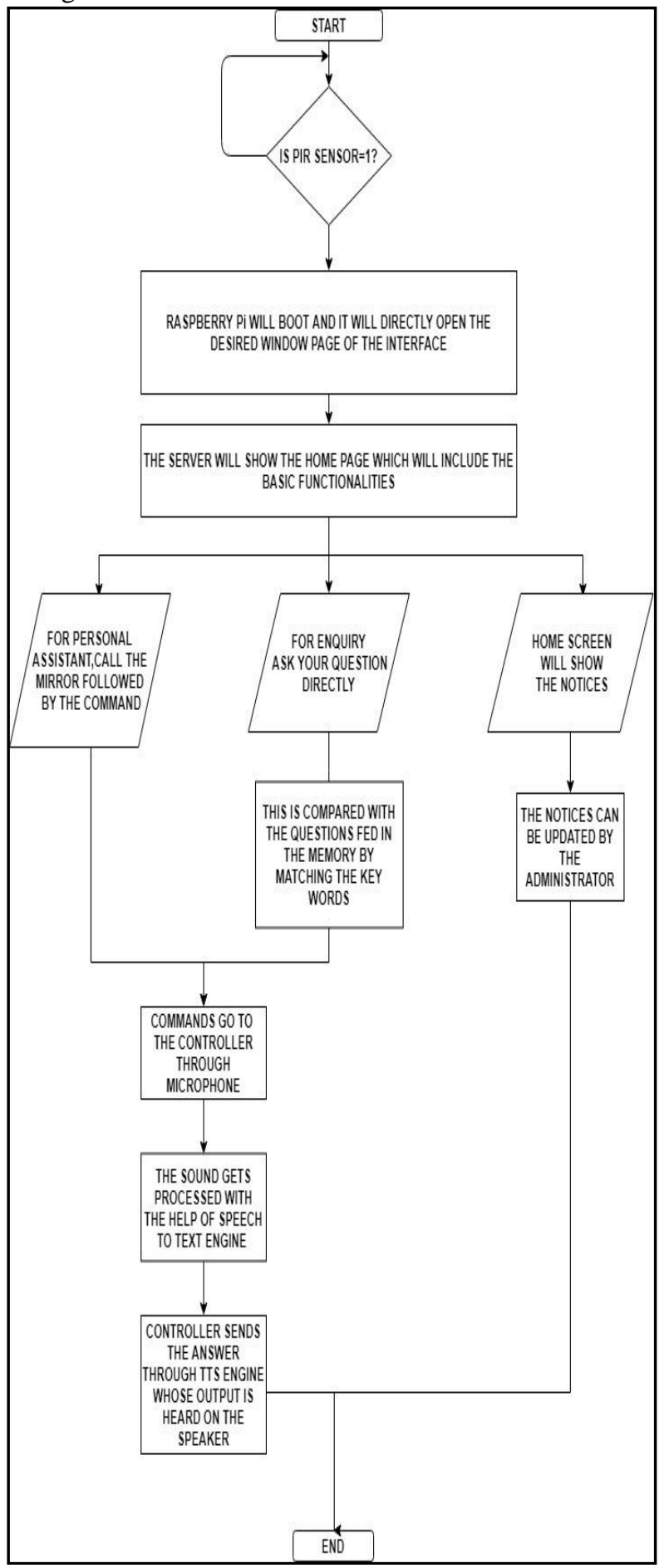

Figure 2 - Workflow diagram.

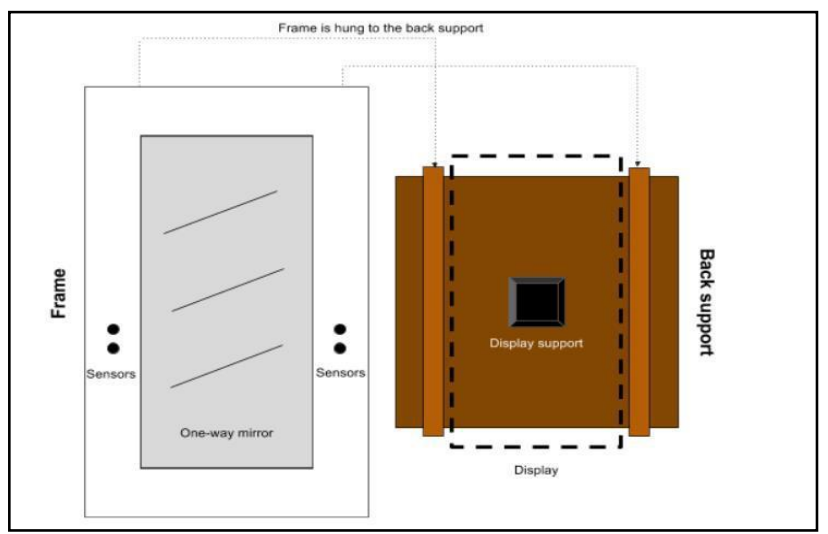

Figure 3-Hardware design

\section{One-way mirror}

The glass used at the front end of the box is probably the most important part of the device or hardware as it is this that is responsible for creating the futuristic and artistic effect and is the biggest part of the smart mirror. Here for it to attain the qualities of reflection and refraction, a dark background surface is needed in which light parts or portions will be visible normally.

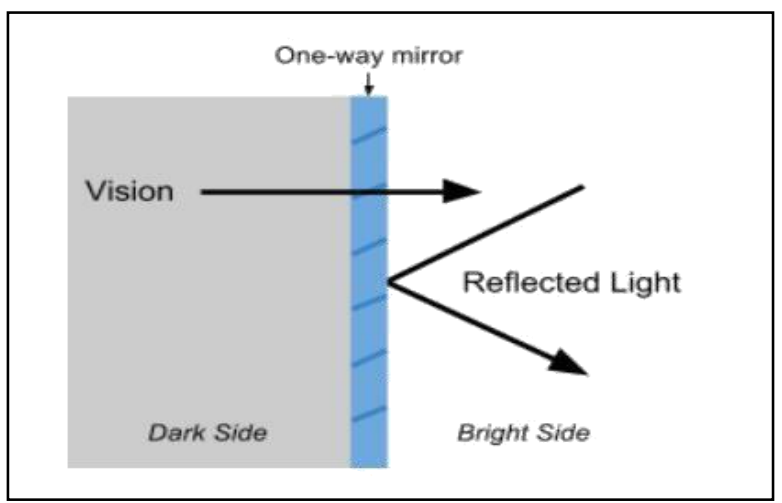

Figure 4-One-way mirror

\section{Display}

For the display a Dell monitor is used, comes with a remote control which is useful to easily turn off the device's screen. The monitor is much smaller than the mirror so a black sticker is used to cover the parts of the glass which are not covered by the display. An HDMI to VGA cable was used to connect the display to the Raspberry Pi for video and audio.

\section{Raspberry Pi}

The Raspberry Pi is a single board computer developed by the Raspberry Pi foundation in the UK. The Pi does not work out of the box. It lacks a hard drive and it does not come with a preinstalled operating system. To install an OS microSD card prepared with an OS image is needed. And because the software that runs on the mirror is coded on the same device at least a screen, a keyboard and a mouse are required.

\section{Microphones and Speakers}

One mode of interaction with the smart mirror is through microphones. USB microphones is used because the Raspberry Pi does not have a regular microphone input. 


\section{Smart Mirror}

Speaker can easily be connected with the output jack port of the raspberry pi

\section{RESULTS}

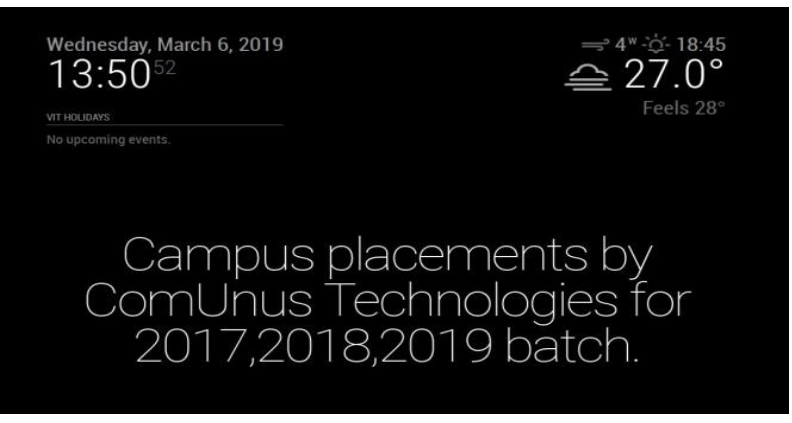

Figure 5-Home screen

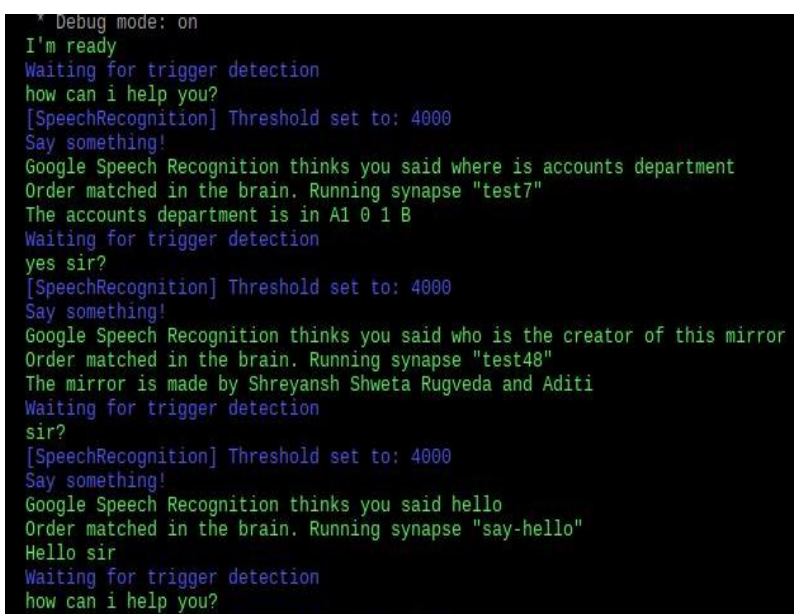

Figure 6- Voice interaction

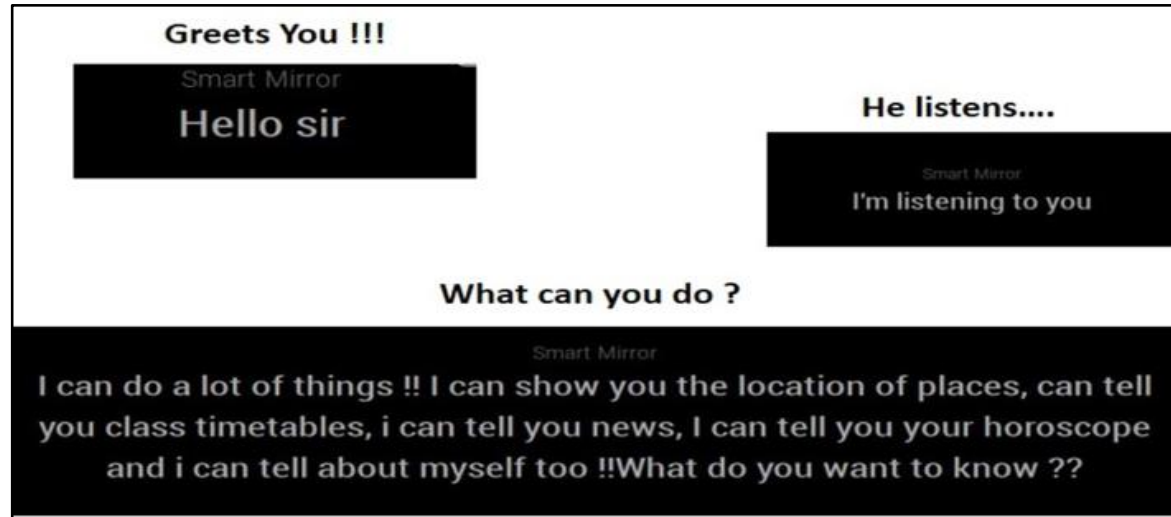

You can ask places..

electronics department is in F block first floor room number F1 04

principal's office is in A block second floor room number A2 02

Ask mirror about himself..

I am a two-way mirror with an electronic display behind the glass. The

display can show the viewer different kinds of information in the form

of widgets, such as weather, time, date, and news updates.

What about me? Do you want to know about my developers?

He can tell you news.

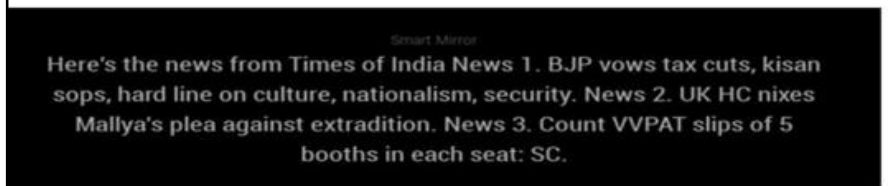

And Horoscope also..

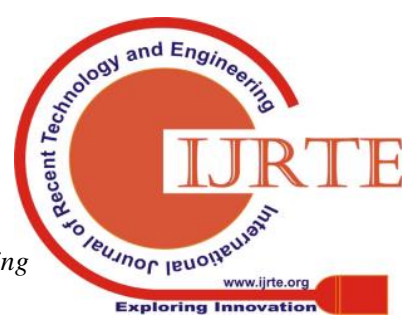




\section{CONCLUSION}

The smart mirror which acts as a smart home control platform is a futuristic system that provides users with an easy-to-use mirror interface, allowing users access to customizable services in a highly interactive manner, while performing other tasks simultaneously. The main strengths are that this is a new kind of smart device that people don't see every day and it looks very spectacular. The mirror works both as a normal mirror as well as a mirror showing daily notifications to the authorized user. There are lots of feeds or notifications that the user can view on the mirror like Facebook, Gmail, news etc. The mirror is also used to display time, weather, date etc. The mirror also acts as a personal assistant as well as displays important notices and is also an enquiry center. The user can interact with the mirror using voice commands. Also, a PIR sensor is attached which turns on the screen only when the user is in the proximity range of the mirror. This reduces power wastage. Smart mirror design has the advantages of small size, simple operation, low cost, high degree of user friendly, personalized user interface and many other advantages which is suitable for many applications like college, home, offices etc. Overall, the proposed smart mirror system incorporates various functionalities to grant users access to personalized information services.

\section{FUTURE SCOPE}

Nothing is perfect and complete and there is always a scope of improvement in each and every product. Everything needs to be updated or upgraded on a timely basis to cope up with the current technology. Apart from up gradation there can be many other features as well which could add up to the proficiency and ability of our smart mirror. There are many future scopes for this paper and hopefully it will emerge into biggest benefit in the field of artificial intelligence. The most basic feature can be smart mirror-based home automation which will provide a natural means of interaction by which we can control the household appliances like switch on/off light and fans through basic voice commands. Majorly, since we are using this mirror in college environment, basic functionalities like barcode scanner or finger print sensor can be integrated to fulfill basic tasks such as college attendance or program registrations etc. This could include registering in programs by scanning of ID cards.

\section{REFERENCES}

1. Raspberry Pi. (2019). Magic Mirror - Raspberry Pi. [online] Available at: Micheal Teaw's official website. [Accessed 17 Jan. 2019].

2. GitHub. (2019). MichMich/MagicMirror. Available at: Mirror Forums [Accessed 17 Jan. 2019].

3. Smart-mirror.io. (2019). Smart Mirror by evancohen. [online] Available at: http://smart-mirror.io/ [Accessed 17 Jan. 2019].

4. Medium. (2019). My Bathroom Mirror Is Smarter Than Yours - Max Braun - Medium. [online] Available at: https://medium.com/@ maxbraun/my-bathroom-mirror-is -smarter-than-yours-94b21c6671ba\#.q4932hjfc

[Accessed 17 Jan. 2019].

5. howchoo. (2019). Build a voice-controlled DIY Raspberry Pi smart mirror with Jasper. [online] [Accessed 17 Jan. 2019].
6. 'How to make a smart mirror' by Hacker Shack https://www.youtube.com/watch?v=fkVBAcvbrjU\&vl=e $\mathrm{n}$ [online] [Accessed 17 Jan. 2019].

7. 'http://api.openweathermap.org/data/' 